Case Report

\title{
Imported Asymptomatic Bancroftian Filariasis Discovered from a Plasmodium vivax Infected Patient: A Case Report from Singapore
}

\author{
Jean-Marc Chavatte ${ }^{1}$ and Roland Jureen ${ }^{2}$ \\ ${ }^{1}$ Malaria Reference Centre, National Public Health Laboratory, Ministry of Health, 3 Biopolis Drive, \\ Synapse 05-14/16, Singapore 138623 \\ ${ }^{2}$ Department of Laboratory Medicine, National University Hospital, 5 Lower Kent Ridge Road, Singapore 119074
}

Correspondence should be addressed to Jean-Marc Chavatte; jean-marc_chavatte@moh.gov.sg

Received 17 April 2017; Accepted 1 June 2017; Published 18 July 2017

Academic Editor: Paul Horrocks

Copyright (C) 2017 Jean-Marc Chavatte and Roland Jureen. This is an open access article distributed under the Creative Commons Attribution License, which permits unrestricted use, distribution, and reproduction in any medium, provided the original work is properly cited.

\begin{abstract}
Human lymphatic filariasis is a vector-borne disease mainly caused by the parasitic nematode Wuchereria bancrofti and transmitted worldwide within the tropical and subtropical regions. Singapore was once endemic for bancroftian filariasis but recent reports are scarce and the disease is nearly forgotten. The case report presented here reports the incidental hospital laboratory finding of an asymptomatic microfilaremia in a relapsing Plasmodium vivax imported case during a malaria treatment follow-up appointment. The parasite was identified by microscopy as $W$. bancrofti and retrospective investigation of the sample collected during malaria onset was found to be also positive. Additional confirmation was obtained by DNA amplification, sequencing, and phylogenetic analysis of the mitochondrial coxl gene that further related the parasite to $W$. bancrofti strains from the Indian region. Considering the large proportion of asymptomatic filariasis with microfilaremia, the high number of migrants and travellers arriving from the surrounding endemic countries, and the common presence of local competent mosquito vectors, Singapore remains vulnerable to the introduction, reemergence, and the spread of lymphatic filariasis. This report brings out from the shadow the potential risk of lymphatic filariasis in Singapore and could help to maintain awareness about this parasitic disease and its public health importance.
\end{abstract}

\section{Introduction}

Lymphatic filariasis (LF) is a mosquito-borne disease caused by nematode parasites of the Family Filarioidea, namely, Wuchereria bancrofti (Cobbold, 1877), Brugia malayi (Brug, 1927), and Brugia timori (Partono et al., 1977). This disease is endemic in the tropical and subtropical areas of Africa, Asia, and Central and South America. Human LF is mainly caused by $W$. bancrofti that is wide spread across these regions and accounts for $90 \%$ of the cases, while the remainder is essentially imputable to B. malayi only present in Asia and Southeast Asia and in a minor proportion to B. timori which is restricted to Timor and Lesser Sunda islands [1]. Humans are the exclusive definitive host for $W$. bancrofti in opposition to $B$. malayi which can be found in human, monkeys, and felines [1]. The zoonotic potential of the filaria from the genus
Brugia is known since Rosenblatt et al. [2] through multiple reports of sporadic cases in US [3-7], Colombia [8], Peru [9], Ethiopia [10], and possibly Indonesia [11] and Malaysia $[12,13]$.

In Singapore, endemic foci of LF due to W. bancrofti were reported since the late 50s [14-18]. At that time, the incidence of LF was $5.5 \%$ in a survey of 902 randomly sampled hospital patients [14]. The microfilarial rates among the major ethnic groups were $4.2 \%, 6.8 \%$, and $5.7 \%$ for the Chinese, Indian, and Malays, respectively [14]. The residential history of the patients indicated an endemic origin of the infection in about $30 \%$ of the Indian and $80 \%$ of the Chinese and Malay [14]. Culex fatigans was incriminated as the main vector since $1.6 \%$ of the 1152 wild-caught mosquitos were carrying larvae of the parasite [14]. While clinical LF was reported as not common [18], Singapore was endemic for LF, which was considered 
as a potential public health problem and representatives from Singapore decided to attend the first Interregional Seminar on Filariasis organized by the WHO in 1965 [19]. Subsequently, less than a handful of studies reported local LF in Singapore: Colbourn and $\mathrm{Ng}$ [20] after having reported 129 confirmed cases (both local and imported) from two hospital records from 1963 to 1967 performed a survey in some selected areas in 1968-1969 and found a microfilarial rate of $1.9 \%$ among mosquito vector and human populations and presence of asymptomatic carriers; Beaver and Cran [21] reported a Wuchereria-like parasite from a soldier returning from service in Singapore; Ho et al. [22] detected filarial antibody by indirect fluorescent antibody technique in 90 amicrofilaraemic sera of patients among a cohort of 324 patients with clinical symptoms suggestive of LF and reported an unequal prevalence among ethnic groups (Indians 48\%, Malays 36\%, and Chinese $15 \%$ ).

Surrounded by LF endemic countries, Singapore remains vulnerable to the introduction of bancroftian filariasis, especially due to the natural presence of competent vectors and the large number of foreigners arriving from LF endemic countries as well as the local travellers. In Malaysia for comparison, LF cases due to $W$. bancrofti were reported among foreign immigrants [17] and became recently more numerous than the local LF cases due to $B$. malayi in Malaysia [23]. Surprisingly, no similar observation is made from Singapore where recent reports of imported LF cases are scarce [24]. This could witness the usual difficulties encountered to confirm LF, as the diagnosis mainly relies on the microscopical observation of microfilaria (MF) that become usually detectable at night time and also as the disease takes months to years to become symptomatic or even may remain asymptomatic with or without microfilaraemia. Interestingly, it is worth noting that the recent case reported by Chew and Teh [24] was based on ultrasound (US) and the observation of the filarial dance sign (FDS), an alternative diagnosis method for LF, commonly used in India [25] and not on the detection of MF in blood.

An unusual case of nephrotic syndrome associated with lymphatic filariasis was also reported without detail about the potential origin of the infection by Yap et al. [26].

The present report contributes to enlightening LF in Singapore and describing the incidental detection of an asymptomatic LF case with presence of MF caused by $W$. bancrofti from an imported relapsing Plasmodium vivax malaria patient.

\section{Case Presentation}

2.1. Onset of Symptoms: A Malaria Case. A 24-year-old Indian male patient who arrived in Singapore from Mumbai, India, in October 2012 for employment presented at the National University Hospital (NUH) in May 2013 with a history of high grade fever associated with chills and rigor the last 4 days. Physical examination was unremarkable except for the presence of fever. The history and clinical examination suggested possible malaria infection with dengue fever as a differential diagnosis and specific laboratory tests were requested. Full blood count revealed thrombocytopenia at
$24 \times 10^{9} /$ L (references: $132-372 \times 10^{9} / \mathrm{L}$ ) and lymphopenia at $0.33 \times 10^{9}$ (references: $0.94-3.08 \times 10^{9} / \mathrm{L}$ ) but was otherwise normal. G6PD was normal and Dengue IgM/IgG/NS1 were negative. Malaria microscopy was positive. The test results thus confirmed the initial suspicion of Plasmodium infection and identified the parasites as Plasmodium vivax with a parasitemia of $0.4 \%$.

Since malaria is a notifiable disease with a surveillance program in Singapore, the case was notified to the Ministry of Health $(\mathrm{MOH})$ and, at the same time, two thin blood films and residual EDTA whole blood were sent for further investigation to the Malaria Reference Centre at the National Public Health Laboratory (MRC-NPHL). During interview the patient declared that he originated from West Bengal, India, and he had an onset of P. vivax malaria in Aug 2012 there prior to his relocation to Singapore. As he was living in a nonmalaria susceptible transmission area of Singapore and based on his declarations the case was classified by $\mathrm{MOH}$ as an imported relapsing case. Morphological and molecular tests for routine malaria surveillance were performed in MRC-NPHL as described previously [27]. Both methods were congruent and confirmed the P. vivax infection. In the meantime, the patient was treated in NUH with chloroquine $600 \mathrm{mg}$ stat, followed by 3 doses of $300 \mathrm{mg} 6 \mathrm{~h}, 24 \mathrm{~h}$, and $48 \mathrm{~h}$ after to cure his malaria infection and the fever lysed and the parasite count rapidly dropped to $0.05 \%$. The patient was discharged the day after admission with planned out-patient follow-up.

2.2. Antimalarial Treatment Follow-Up: A Filaria Case. During the follow-up appointment in NUH in June 2013, the patient was asymptomatic and a thick blood film was prepared and found negative for malaria but unexpectedly showed the presence of several microfilaria. Intrigued and surprised by this finding and also uncertain about the morphological features to identify this parasite, the hospital laboratory staffs sent the thick film and residual of whole EDTA blood to the MRC-NPHL. Microscopist prepared additional blood films stained with Giemsa according to standard procedure [28] and protected them with coverslip mounted with Eukitt ${ }^{\circledR}$ (Sigma-Aldrich). The blood films were entirely screened at low magnification (100x) to detect the MF that were studied in detail at higher magnifications $(\times 400$ to $\times 1000$ ) with an Olympus CX31 microscope (Olympus). Morphometric measurement and microphotographs were taken with a Nikon Eclipse 80i microscope equipped with a Nikon DS Ril camera and Nikon NIS Element D Imaging Software (Nikon).

The parasites illustrated in Figures $1(\mathrm{a})-1(\mathrm{k})$ were identified as MF of Wuchereria bancrofti based on their morphological characteristics such as presence of sheath unstained or lightly stained with Giemsa stain (Figures 1(a), 1(c), and $1(\mathrm{j})$ ), cephalic space proportions (Figures $1(\mathrm{a})-1(\mathrm{c}), 1(\mathrm{e})$, and 1(f)), and tail that tapers to delicate point without nucleus at the tip (Figures 1(a), 1(d), 1(e), 1(i), and 1(j)) as well as morphometric measurements: $272.9 \pm 16.4 \times 6.6 \pm 1.2 \mu \mathrm{m}$ (range 234.6-292.57 $\times 4.57-8.34 \mu \mathrm{m} ; n=10$ ) for length and width (three points' measurement), respectively. 


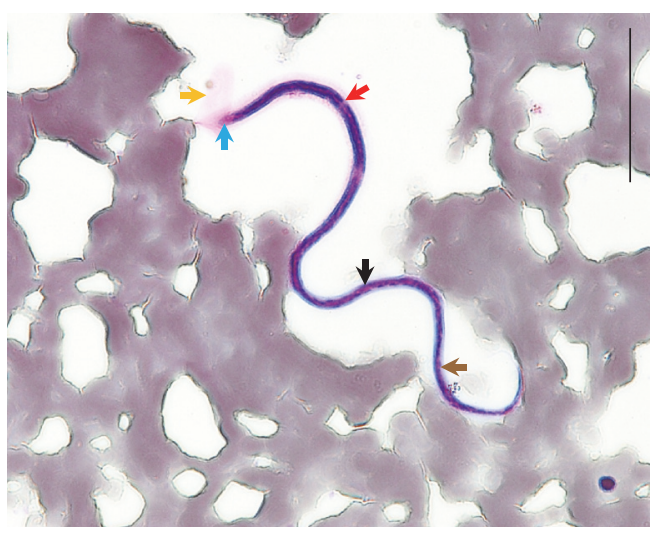

(a)

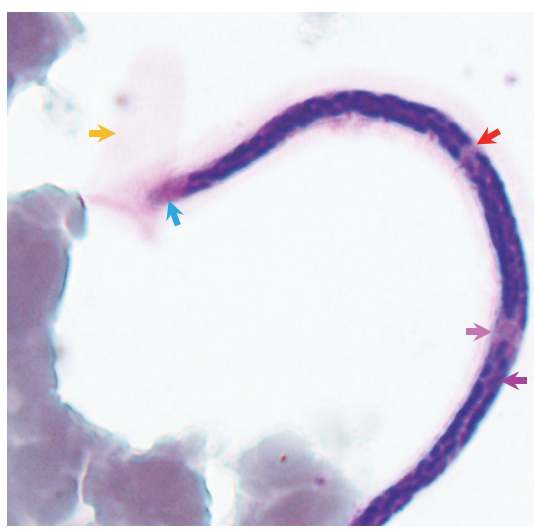

(c)

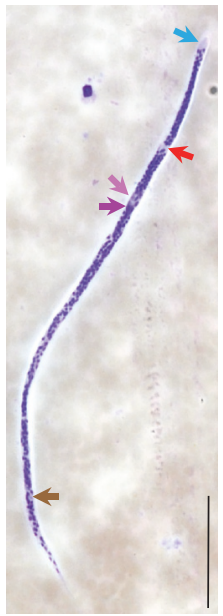

(e)

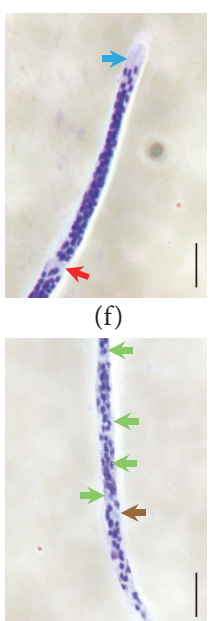

(h)

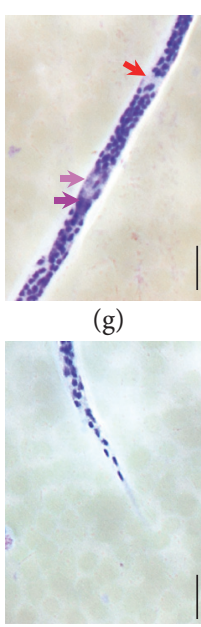

(i)

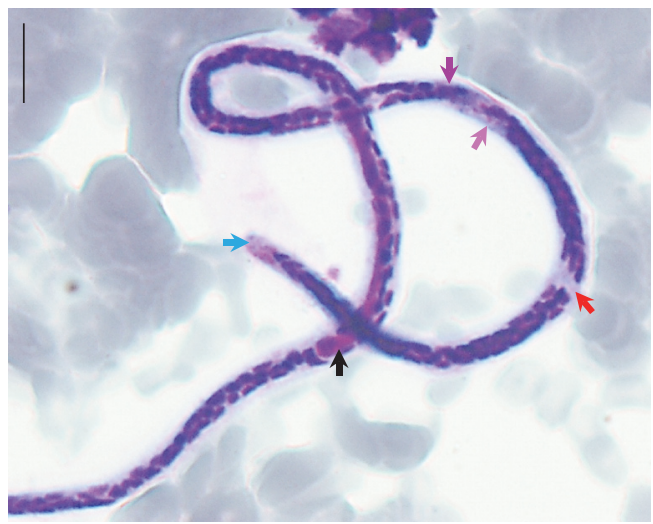

(b)

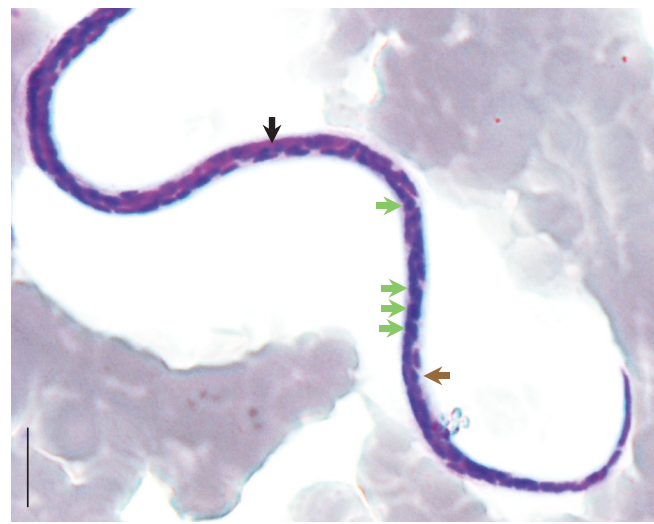

(d)

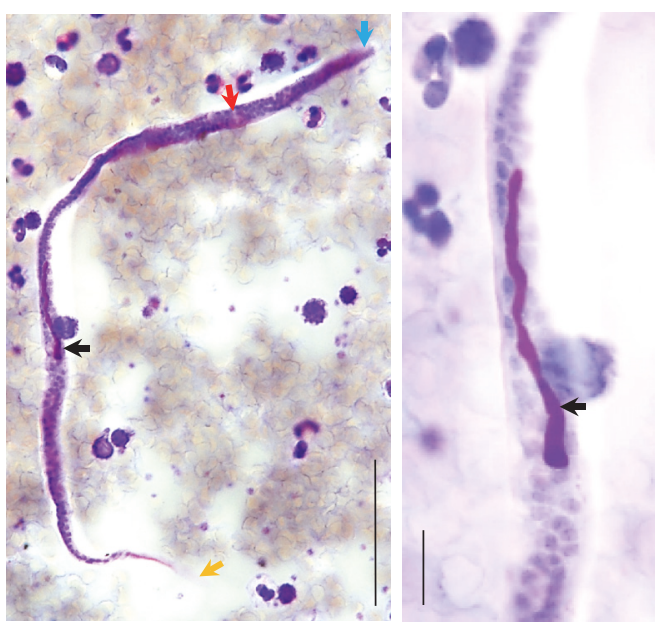

(k)

Figure 1: Microphotographs of Wuchereria bancrofti microfilaria in blood smears. (a-d) Thin blood films; (e-k) thick blood films; (a, e, j) full size microfilaria; (b) head and body details; (c, f) head details; (d) body and tail details; (g, h) body details; (i) tapered tail tip details free of terminal nuclei; (k) detail of the inner body. Coloured arrows represent sheath (orange), cephalic space (blue), nerve ring (red), excretory pore (pink), excretory cell (purple), inner body (black), germinal cells (green), and anal pore (brown) across the different pictures. Scale bars: $(\mathrm{a}, \mathrm{e}, \mathrm{j})=50 \mu \mathrm{m} ;(\mathrm{b}-\mathrm{d}, \mathrm{f}-\mathrm{i}, \mathrm{k})=10 \mu \mathrm{m}$.

As alternative confirmatory approach, the sample was subjected to molecular testing. DNA was extracted and stored as described previously [27]. Amplification of the filarial parasite DNA was carried out by polymerase chain reaction (PCR) targeting the coxl and the $12 S$ rRNA genes from the parasite mitochondrion according to the protocols developed by Casiraghi et al. [29]. PCRs were run on Veriti ${ }^{\circledR}$ Thermal Cycler (Applied Biosystem ${ }^{\circledR}$ ) and the PCR products were visualized after electrophoresis performed with the QIAxcel ${ }^{\circledR}$ Advanced instrument $\left(\right.$ Qiagen ${ }^{\circledR}$ ) equipped with 
QIAxcel DNA Screening Kit (Qiagen). The $12 S$ rRNA gene reactions did not generate any results despite repeats. PCR products obtained for the coxl gene were purified using the QIAquick ${ }^{\circledR}$ PCR Purification Kit (Qiagen) and stored at $-30^{\circ} \mathrm{C}$ until usage. Purified PCR products were sequenced in both directions using the appropriate oligonucleotide primers as described previously [27]. Alignment and crosschecking of the sequences were performed with CLC Main Workbench 7.7 software (CLC Bio, Qiagen) and consensus sequences of $655 \mathrm{bp}$ of the coxl gene were obtained. Comparison of the sequences using Basic Local Alignment Tool (BLAST) [30] confirmed the identity of the MF as $W$. bancrofti. The sequence has been deposited in GenBank under the following accession number: KY883763. A multiple sequences alignment of the coxl gene including 37 sequences of common human parasitic roundworms retrieved from GenBank and KY883763 was generated by multiple sequences comparison by log-expectation (MUSCLE) algorithm [31]. A phylogenetic analysis was performed using the Maximum Likelihood (ML) method based on GTR $+\Gamma$ model [32]. The most appropriate model of nucleotide substitution for ML was selected based on Akaike's Information Criterion value [33]. This analysis showed that the coxl sequence of the $W$. bancrofti strain presented here clustered with other sequences from India and Sri Lanka (Figure 2), corroborating the epidemiological data and the idea of an imported parasite.

2.3. Retrospective Investigation of the Malaria Sample. Based on the confirmed presence of $W$. bancrofti MF in the blood of the patient, a retrospective investigation of his $P$. vivax positive blood sample taken a month earlier and archived in MRC-NPHL was initiated. The two original thin blood films provided by NUH laboratory were entirely screened at low magnification and revealed the presence of one MF in each blood film within the thick part of the smear (Figures 1(a) and $1(\mathrm{~b})$ ). This result confirms that the patient was already infected with detectable MF of $W$. bancrofti that were missed out by medical technologists in both NUH and MRC-NPHL as they only focused on the tail of the blood films for the malaria investigations at that time.

2.4. Antifilarial Treatment and Outcome. This laboratory finding was notified to the clinician in charge of the patient who informed him and prescribed a course of Albendazole $(400 \mathrm{mg})+$ Ivermectin $(200 \mu \mathrm{g} / \mathrm{kg})$. The patient responded well to the treatment.

\section{Discussion}

3.1. Local Transmission and Introduction of W. bancrofti. Historically bancroftian filariasis was locally transmitted in Singapore $[14,15,19-22]$. While recent data about LF in Singapore are scarce [24], the present report confirms the detection of $W$. bancrofti from an imported malaria case and further highlights the asymptomatic carriage of this pathogen in a foreign worker. Asymptomatic cases of LF are very common as the disease takes months to years to evolve $[1,34]$ but remain a serious threat for the introduction of the parasite in the nonendemic countries as some are microfilaremic and could potentially become a reservoir and source for the spread of the disease by local competent vectors [34]. Classified as an imported relapsing case for his onset of malaria, the filarial infection of the case presented here is also likely to be acquired before his relocation to Singapore since the patient had only stayed here for around 8 months before this laboratory finding, none of his roommates displayed symptoms, and vector controls were regularly carried out in the vicinity where he stayed. Therefore, our case typically illustrates the situation of asymptomatic carriers of LF, who harbour MF and who could potentially introduce LF into the local community as they may stay over an extended period.

3.2. Diagnosis of Bancroftian Filariasis. W. bancrofti is not always easy to diagnose in clinical laboratories, even in patients with suggestive symptoms, as the diagnosis essentially relies on the microscopic detection of $\mathrm{MF}$ in the blood. In fact, MF have a different periodicity depending of the geographical region from where the parasite originates implying the need for blood collection when MF appears in the bloodstream (usually at night) to render the parasite detectable by the standard thin/thick films microscopy methods [28, 35]. Concentration techniques such as Knott's technique [36], microhematocrit tube, and membrane filtration technique facilitate detection by microscopy but require more steps and are time-consuming [28, 35, 37]. Since the 1990s, alternative tests have emerged providing significant progress in LF diagnosis [38]. Firstly, serological tests that are considered a better alternative than microscopic methods have been developed in two approaches: (i) immunoenzymatic technique detecting antifilarial antibodies (IgG4) that are usually high in patients with active filarial infection [39, 40]; (ii) immunochromatographic tests detecting circulating filarial antigen [41]. These tests have been adapted to rapid diagnostic tests and are regarded as the gold standard due to their simplicity of usage, high sensitivity, and specificity, independency of blood collection time, and their rapidity $[37,38]$. Secondly, molecular methods such as PCR have become available for the detection of $W$. bancrofti DNA from blood samples [42-44] but still remain hardly used in clinical settings. Thirdly, ultrasound methods have been employed. They constitute a noninvasive approach and allow the direct observation of the adult worms movements or fluxes of fluid displaced by their movements and are described as the FDS $[24,25,38,45]$. In our case the diagnosis was made by chance while reviewing a thick blood film for the follow-up of malaria treatment. Despite being large organisms, readily observable, MF were missed twice in the first samples tested for malaria; it seems therefore important to remind clinical laboratory staff to not only focus on the main test requested for a sample but also consider the possibility of coinfection whenever possible. Blood films microscopy is a broad range test that must be carefully reviewed for the presence of any hematozoa that may be present and may greatly vary in size from tiny Babesia trophozoite $(\approx 1 \mu \mathrm{m})$ to large $\mathrm{MF}(>250 \mu \mathrm{m}$ in length).

3.3. MF Periodicity. Based on the time of appearance of the MF in the blood, there is three recognised subtypes of $W$. 


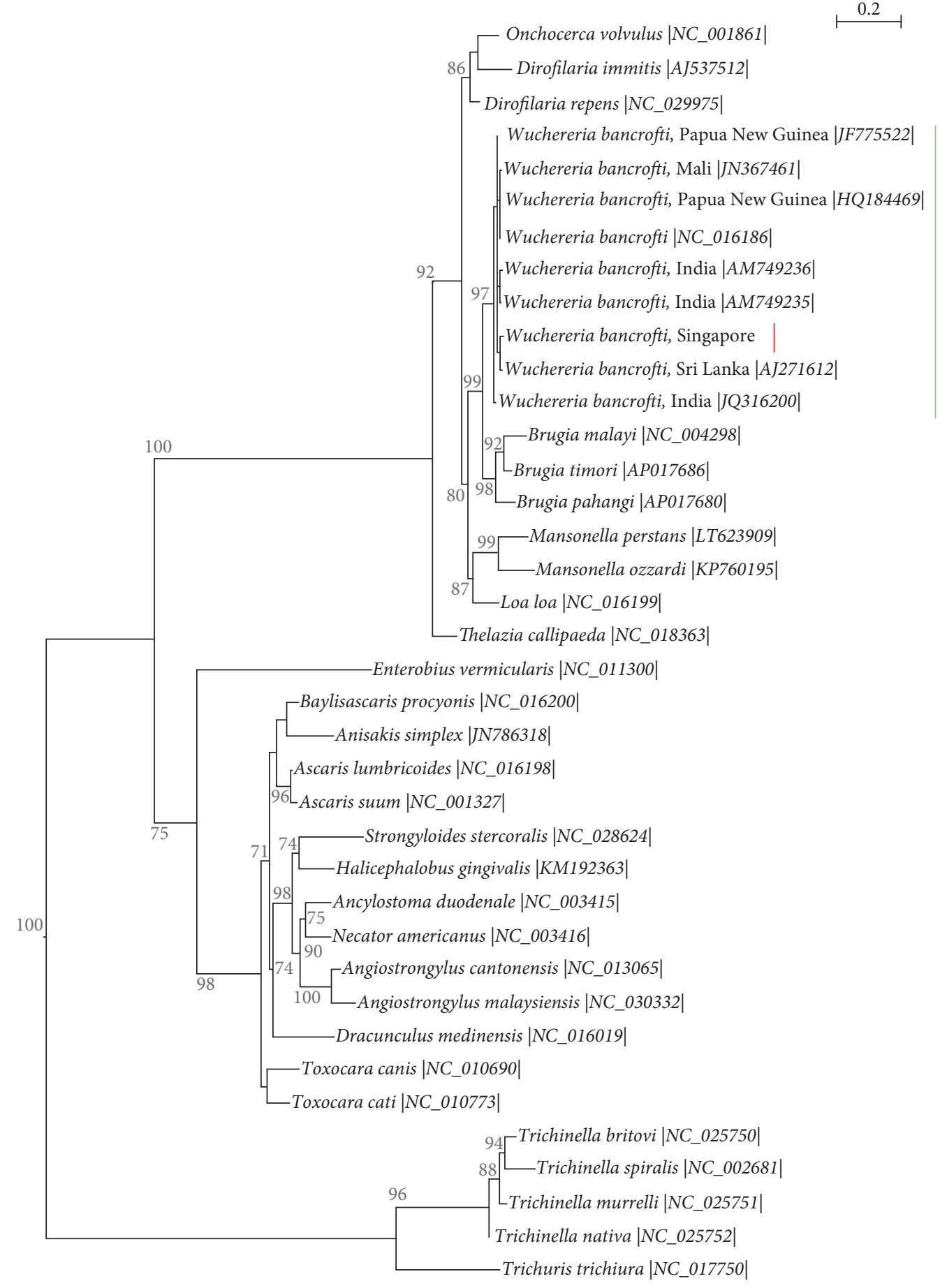

FIGURE 2: Molecular phylogeny of common parasitic human roundworms based on the coxl gene. The analysis is inferred by ML method with GTR $+\Gamma$ model of evolution. It included 37 DNA sequences downloaded from GenBank (accession number provided between vertical bars) and the sequence obtained from the present case. The parasites of the order Trichocephalida serve as outgroup to root the tree. The tree with the highest $\log$ likelihood $(-5385.0252)$ is displayed. One thousand nonparametric bootstrap analyses were used to assess nodal robustness and tree topology reliability, branch support $>70 \%$ only shown. Lines highlight lymphatic filaria (blue); among them are the Wuchereria parasites (green) and within this genus the sequence obtained from the present case (red), respectively. 
bancrofti, namely, the nocturnally periodic, the nocturnally subperiodic, and the diurnally subperiodic types $[46,47]$. These three variants have sometimes been further classified into ecotypes based on their vector preference [46] and showing a perfect adaptation between the parasite and the vector for an optimal transmission [46, 48]. MF periodicity shows some geographical variations mainly related to the presence and biting behaviour of the local competent vectors; for example, the nocturnally periodic strains are primarily vectorised by Culex quinquefasciatus in urban areas of Asia, East Africa, and South and Central America and by different Anopheles species in the rural areas [49]. In the present case, the samples were collected at 18:25 and 9:36 at the admission time and at 14:06 during the follow-up appointment, respectively. As both samples harboured detectable MF, it could be hypothesized that the patient was infected with a nocturnally subperiodic strain whose MF are present in the blood throughout the day and peak at night since diurnally periodic strains are essentially prevalent in Pacific region.

3.4. Public Health Problem. The very low number of LF reports from Singapore [14, 20-22, 24] is questionable and several explanations may concur to it. Firstly, local LF transmission has not been reported over the past 30 years $[14,20-22,24]$ and is likely to be indirectly due to the strong and strict vector control polices set in place to maintain the free malaria status of the county as well as to limit the transmission of the arboviruses, leading to a really low or no occurrence of local filaria transmission in Singapore. Secondly, the low number of imported LF cases [24] is surprising but might be explained by a general overlook of the filaria in our settings. In fact, as mentioned above these parasites are sometimes difficult to diagnose with standard microscopy that is often the only method available in the clinical laboratory $[28,35,37]$. There is also a general lack of awareness about LF that is classified as Neglected Tropical Diseases (NTD) by the World Health Organization (WHO). In the perspective of an increase of the population, with a related increase of human migratory flux (large numbers of foreigners arriving from endemic countries and locals travelling there) LF should not be forgotten and should still be considered as a potential public health threat, particularly due to the natural presence of common competent mosquito vectors. Taking advantage of the present case, this report should help to raise awareness locally among all health related workers about LF.

3.5. Local Vectors of W. bancrofti. There are six mosquito genera, namely, Aedes, Anopheles, Culex, Downsiomyia, Mansonia, and Ochlerotatus, that contain species reported to be vectors of $W$. bancrofti in South East Asia [47, 50]. Among them, C. quinquefasciatus formerly called C. fatigans was proved to be the main local vector in the country $[14,20]$. This mosquito species is also reported as the main vector of LF due to W. bancrofti in urban areas of India [51], Sri Lanka [52], and also Thailand [53] and remains a very common mosquito in the urbanized Singapore city $[54,55]$. Additionally, several other species known to be vectors of bancroftian filariasis in the region such as Anopheles maculatus [56] and Mansonia uniformis [57] are also found in Singapore [54, 55] and may constitute potential secondary vectors.

3.6. Brugian Filariasis. Although there is no report of LF attributable to Brugia spp. in Singapore to date, it should be kept in mind that parasites of this genus are circulating in the neighbour countries. While the risk of transmission of B. timori is extremely low as this species is restricted to Timor and Lesser Sunda islands, the risk of transmission of $B$. malayi and B. pahangi is not negligible. Regarding the human parasite B. malayi, it is the main cause of local LF in Malaysia and has been shown to have an animal reservoir beside its human one that complicates its control [1]. It is mainly transmitted by the mosquito of the genera Mansonia and Anopheles, for example, Mansonia uniformis and Anopheles barbirostris, which are present in Singapore $[54,55]$. Regarding the felid parasite $B$. pahangi, it has recently been reported as a zoonotic pathogen inducing LF with domestic cats as reservoir $[13,58]$ in several transmission events in the suburb of the capital city of Malaysia, Kuala Lumpur $[13,58]$, and in a semirural town of Selangor $[12,59]$. The incriminated vector was Armigeres subalbatus [12, 59], a mosquito species also present in Singapore $[55,60]$.

\section{Data Access}

Genetic sequence obtained from this study is deposited in GenBank (accession number KY883763). The remaining available material consisting of blood films is archived in the MRC-NPHL (numbers FIL13/01 and MAL13/52).

\section{Ethical Approval}

The samples were collected for malaria surveillance purpose for the $\mathrm{MOH}$ under the Infectious Diseases Act (Chapter 137), Part III, Control of Infectious Diseases within Singapore, Section 7, Public Health Surveillance Programmes.

\section{Conflicts of Interest}

The authors declare that there are no conflicts of interest regarding the publication of this paper.

\section{Authors' Contributions}

Jean-Marc Chavatte performed the microscopical and molecular studies. Roland Jureen and Jean-Marc Chavatte drafted the manuscript. Both authors have read and agreed on the last version of the manuscript.

\section{Acknowledgments}

The authors are grateful to the medical technologists of the Haematology Laboratory, Department of Laboratory Medicine, National University Hospital, for the detection of the microfilaria and their help in processing the samples. The study was supported by the National Public Health Laboratory of the Ministry of Health of Singapore. 


\section{References}

[1] World Health Organization, "Lymphatic filariasis: the disease and its control," Fifth Report of The WHO Expert Committee on Filariasis, Geneva, Switzerland, 1992.

[2] P. Rosenblatt, P. C. Beaver, and T. C. Orihel, "A filarial infection apparently acquired in New York City," The American Journal of Tropical Medicine and Hygiene, vol. 11, pp. 641-645, 1962.

[3] P. C. Beaver and T. C. Orihel, "Human infection with filariae of animals in the United States," The American Journal of Tropical Medicine and Hygiene, vol. 14, no. 6, pp. 1010-1029, 1965.

[4] J. K. Baird, L. I. Alpert, and R. Friedman, "North American Brugian filariasis: report of nine infections of humans," The American Journal of Tropical Medicine and Hygiene, vol. 35, no. 6, pp. 1205-1209, 1986.

[5] T. C. Orihel and P. C. Beaver, "Zoonotic Brugia infections in North and South America," The American Journal of Tropical Medicine and Hygiene, vol. 40, no. 6, pp. 638-647, 1989.

[6] T. C. Orihel and M. L. Eberhard, "Cerebral filariasis in rhodesia-a zoonotic infection?" The American Journal of Tropical Medicine and Hygiene, vol. 22, no. 5, pp. 596-599, 1973.

[7] A. E. Paniz-Mondolfi, T. Gárate, C. Stavropoulos et al., "Zoonotic filariasis caused by novel Brugia sp. nematode, United States, 2011," Emerging Infectious Diseases, vol. 20, no. 7, pp. 1248-1250, 2014.

[8] W. J. Kozek, M. A. Reyes, J. Ehrman, F. Garrido, and M. Nieto, "Enzootic Brugia infection in a two-year-old Colombian girl," American Journal of Tropical Medicine and Hygiene, vol. 33, no. 1, pp. 65-69, 1984.

[9] J. K. Baird and R. C. Neafie, "South American Brugian filariasis: report of a human infection acquired in Peru," American Journal of Tropical Medicine and Hygiene, vol. 39, no. 2, pp. 185-188, 1988.

[10] M. C. Menéndez and M. Bouza, "Brugia species in a man from western ethiopia," The American Journal of Tropical Medicine and Hygiene, vol. 39, no. 2, pp. 189-190, 1988.

[11] J. R. Palmieri, S. Ratiwayanto, S. Masbar, S. Tirtokusumo, J. Rusch, and H. A. Marwoto, "Evidence of possible natural infections of man with Brugia pahangi in south Kalimantan (Borneo), Indonesia," Tropical and Geographical Medicine, vol. 37, no. 3, pp. 239-244, 1985.

[12] A. Muslim, Filariasis in Kuala Lumpur and Selangor: Entomological, Parasitological and Molecular Studies, University of Malaya, Kuala Lumpur, Malaysia, 2010.

[13] L. H. Tan, M. Y. Fong, R. Mahmud, A. Muslim, Y. L. Lau, and A. Kamarulzaman, "Zoonotic Brugia pahangi filariasis in a suburbia of Kuala Lumpur City, Malaysia," Parasitology International, vol. 60, no. 1, pp. 111-113, 2011.

[14] T. J. Danaraj, J. F. Schacher, and D. H. Colless, "Filariasis in Singapore," The Medical journal of Malaya, vol. 12, no. 4, pp. 605-612, 1958.

[15] T. Wilson, "Filariasis in Malaya-A general review," Transactions of the Royal Society of Tropical Medicine and Hygiene, vol. 55, no. 2, pp. 107-129, 1961.

[16] J. F. Edeson and T. Wilson, "The Epidemiology of filariasis due to Wuchereria bancrofti and Brugia malayi," Annual Review of Entomology, vol. 9, no. 1, pp. 245-268, 1964.

[17] A. A. Sandosham, "Review of research in parasitology in Malaya," Singapore Medical Journal, vol. 4, pp. 42-51, 1963.

[18] R. S. Desowitz, "Review of research in parasitology in Singapore," Singapore Medical Journal, vol. 4, pp. 30-33, 1963.
[19] J. F. Kessel, "Filariasis as a world problem," in Mosquito News, pp. 490-497, 1966.

[20] M. J. Colbourn and W. K. Ng, "An assessment of filariasis transmission in Singapore," Southeast Asian Journal of Tropical Medicine and Public Health, vol. 3, pp. 40-44, 1972.

[21] P. C. Beaver and I. R. Cran, "Wuchereria like filaria in an artery, associated with pulmonary infarction," American Journal of Tropical Medicine and Hygiene, vol. 23, no. 5, pp. 869-876, 1974.

[22] L. C. Ho, M. Singh, E. H. Yap, and B. C. Ho, "Studies on filariasis in Singapore: immunodiagnosis using indirect immunofluorescence," Southeast Asian Journal of Tropical Medicine and Public Health, vol. 15, pp. 175-178, 1984.

[23] I. Vythilingam, "Plasmodium knowlesi and Wuchereria bancrofti: Their vectors and challenges for the future," Frontiers in Physiology, vol. 3, article 115, 2012.

[24] L.-L. Chew and H.-S. Teh, "The filarial dance sign in scrotal filarial infection: A case report," Journal of Clinical Ultrasound, vol. 41, no. 6, pp. 377-379, 2013.

[25] S. Suresh, V. Kumaraswami, I. Suresh et al., "Ultrasonographic diagnosis of subclinical filariasis," Journal of Ultrasound in Medicine, vol. 16, no. 1, pp. 45-49, 1997.

[26] H. K. Yap, K. T. Woo, P. P. B. Yeo, G. S. C. Chiang, M. T. Singh, and C. Lim, "The nephrotic syndrome associated with filariasis," Annals of the Academy of Medicine, Singapore, vol. 11, pp. 61-63, 1982.

[27] J.-M. Chavatte, S. B. H. Tan, G. Snounou, and R. T. P. V. Lin, "Molecular characterization of misidentified Plasmodium ovale imported cases in Singapore," Malaria Journal, vol. 14, no. 1, article no. 454, 2015.

[28] World Health Organization, Manual of Basic Techniques for a Health Laboratory, Geneva, Switzerland, 2nd edition, 2003.

[29] M. Casiraghi, T. J. C. Anderson, C. Bandi, C. Bazzocchi, and C. Genchi, "A phylogenetic analysis of filarial nematodes: Comparison with the phylogeny of Wolbachia endosymbionts," Parasitology, vol. 122, no. 1, pp. 93-103, 2001.

[30] S. F. Altschul, W. Gish, W. Miller, E. W. Myers, and D. J. Lipman, "Basic local alignment search tool," Journal of Molecular Biology, vol. 215, no. 3, pp. 403-410, 1990.

[31] R. C. Edgar, "MUSCLE: multiple sequence alignment with high accuracy and high throughput," Nucleic Acids Research, vol. 32, no. 5, pp. 1792-1797, 2004.

[32] M. Nei and S. Kumar, Molecular Evolution and Phylogenetics, Oxford University Press, 2000.

[33] H. Akaike, "A New Look at the Statistical Model Identification," IEEE Transactions on Automatic Control, vol. 19, no. 6, pp. 716723, 1974.

[34] S. Babu and T. B. Nutman, "Immunopathogenesis of lymphatic filarial disease," Seminars in Immunopathology, vol. 34, no. 6, pp. 847-861, 2012.

[35] World Health Organization, "Lymphatic filariasis," Fourth report of the WHO Expert Committee on Filariasis, Geneva, Switzerland, 1984.

[36] J. Knott, "A method for making microfilarial surveys on day blood," Transactions of the Royal Society of Tropical Medicine and Hygiene, vol. 33, no. 2, pp. 191-196, 1939.

[37] S. Phantana, S. Sensathein, J. Songtrus, S. Klagrathoke, and K. Phongnin, "ICT filariasis test : A new screening test for Bancroftian filariasis," Southeast Asian Journal of Tropical Medicine and Public Health, vol. 30, no. 1, pp. 47-51, 1999. 
[38] A. Rocha, C. Braga, M. Belém et al., "Comparison of tests for the detection of circulating filarial antigen (Og4C3-ELISA and AD12-ICT) and ultrasound in diagnosis of lymphatic filariasis in individuals with microfilariae," Memorias do Instituto Oswaldo Cruz, vol. 104, no. 4, pp. 621-625, 2009.

[39] G. E. Kwan-Lim, K. P. Forsyth, and R. M. Maizels, "Filarialspecific IgG4 responses correlates with active Wuchereria infection," Journal of Immunology, vol. 145, pp. 4298-4305, 1990.

[40] A. Kurniawan, M. Yazdanbakhsh, R. Van Ree et al., "Differential expression of IgE and IgG4 specific antibody responses in asymptomatic and chronic human filariasis," Journal of Immunology, vol. 150, no. 9, pp. 3941-3950, 1993.

[41] G. J. Weil, P. J. Lammie, and N. Weiss, "The ICT filariasis test: A rapid-format antigen test for diagnosis of bancroftian filariasis," Parasitology Today, vol. 13, no. 10, pp. 401-404, 1997.

[42] J. S. McCarthy, M. Zhong, R. Gopinath, E. A. Ottesen, S. A. Williams, and T. B. Nutman, "Evaluation of a polymerase chain reaction-based assay for diagnosis of Wuchereria bancrofti infection," Journal of Infectious Diseases, vol. 173, no. 6, pp. 1510 $1514,1996$.

[43] M. Zhong, J. McCarthy, L. Bierwert et al., "A polymerase chain reaction assay for detection of the parasite Wuchereria bancrofti in human blood samples," American Journal of Tropical Medicine and Hygiene, vol. 54, no. 4, pp. 357-363, 1996.

[44] R. M. R. Ramzy, H. A. Farid, I. H. Kamal et al., "A polymerase chain reaction-based assay for detection of Wuchereria bancrofti in human blood and Culex pipiens," Transactions of the Royal Society of Tropical Medicine and Hygiene, vol. 91, no. 2, pp. 156160, 1997.

[45] F. Amaral, G. Dreyer, J. Figueredo-Silva et al., "Live adult worms detected by ultrasonography in human bancroftian filariasis," American Journal of Tropical Medicine and Hygiene, vol. 50, no. 6, pp. 753-757, 1994.

[46] M. Sasa, in Human Filariasis: A Global Survey of Epidemiology and Control, p. 819, University Park Press, London, UK, 1976.

[47] J. W. Mak, "Epidemiology of lymphatic filariasis," Ciba Foundation Symposium, vol. 127, pp. 5-14, 1987.

[48] M. V. Weerasooriya, M. P. Mudalige, N. K. Gunawardena, E. Kimura, and W. A. Samarawickrema, "Microfilarial periodicity of Wuchereria bancrofti and man landing periodicity of the vector Culex quinquefasciatus say in Matara, Sri Lanka," THe Ceylon Medical Journal, vol. 43, no. 2, pp. 78-83, 1998.

[49] M. J. Bockarie, E. M. Pedersen, G. B. White, and E. Michael, "Role of vector control in the global program to eliminate lymphatic filariasis," Annual Review of Entomology, vol. 54, pp. 469-487, 2009.

[50] N. Zagaria and L. Savioli, "Elimination of lymphatic filariasis: a public-health challenge," Annals of Tropical Medicine and Parasitology, vol. 96, no. 8, pp. 3-13, 2002.

[51] P. K. Das, A. Manoharan, S. Subramanian et al., "Bancroftian filariasis in Pondicherry, South India - epidemiological impact of recovery of the vector population," Epidemiology and Infection, vol. 108, no. 3, pp. 483-493, 1992.

[52] F. P. Amerasinghe and N. B. Munasingha, "A predevelopment mosquito survey in the Mahaweli Development Project area, Sri Lanka: adults.," Journal of Medical Entomology, vol. 25, no. 4, pp. 276-285, 1988.

[53] S. Triteeraprapab, K. Kanjanopas, S. Suwannadabba, S. Sangprakarn, Y. Poovorawan, and A. L. Scott, "Transmission of the nocturnal periodic strain of Wuchereria bancrofti by Culex quinquefasciatus: Establishing the potential for urban filariasis in Thailand," Epidemiology and Infection, vol. 125, no. 1, pp. 207212, 2000.

[54] C. Apiwathnasorn, "A list of mosquito species in Southeast Asia," in Museum and Reference Centre, p. 73, Museum and Reference Centre, SEAMEO-TROPMED National Centre of Thailand, Mahidol University, 1986.

[55] National Environmental Agency, "Some common mosquitoes and nuisance insects in Singapore," p. 30, Singapore, 2008.

[56] W. H. Cheong and A. H. Omar, "Anopheles maculatus, a new vector of Wuchereria bancrofti in Malaysia (Pulau Aur) and a potential vector on mainland Malaya," The Medical Journal of Malaya, vol. 20, no. 1, pp. 74-75, 1965.

[57] H. De Rook, in Filariasis on the Island of Pam, pp. 197-212, South Waigeo District, West New-Guinea, 1957.

[58] A. Muslim, M. Y. Fong, R. Mahmud, and S. Sivanandam, "Vector and reservoir host of a case of human Brugia pahangi infection in Selangor, peninsular Malaysia," Tropical Biomedicine, vol. 30, no. 4, pp. 727-730, 2013.

[59] A. Muslim, M.-Y. Fong, R. Mahmud, Y.-L. Lau, and S. Sivanandam, "Armigeres subalbatus incriminated as a vector of zoonotic Brugia pahangi filariasis in suburban Kuala Lumpur, Peninsular Malaysia," Parasites and Vectors, vol. 6, no. 1, article 219, 2013.

[60] J. Jeffery, R. M. L. Lee, S. Y. Tan, C. Liew, L. C. Ng, and S. G. LamPhua, "New mosquito species records (Diptera: Culicidae) from Singapore," Tropical Biomedicine, vol. 27, no. 1, pp. 138-142, 2010. 


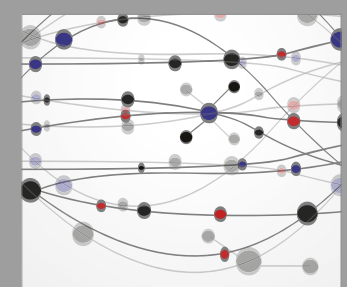

The Scientific World Journal
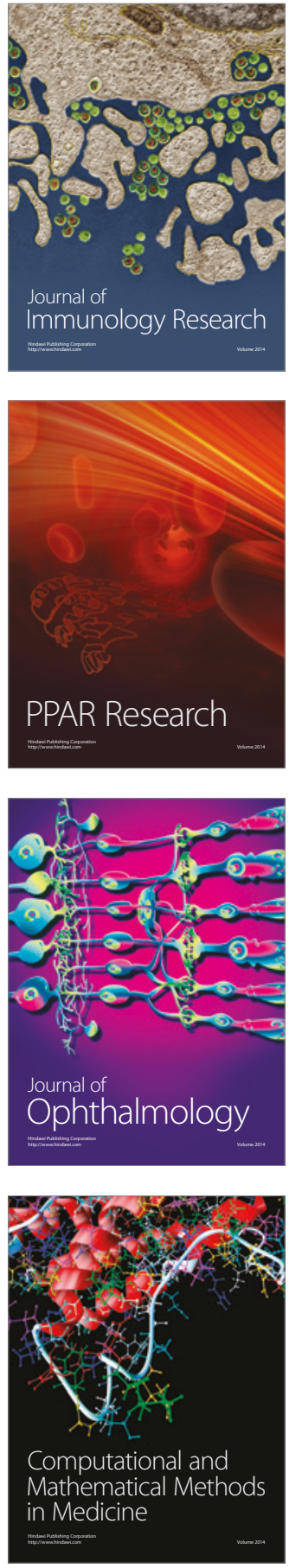

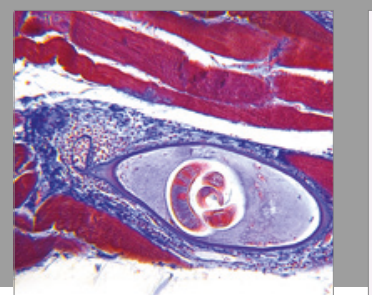

Gastroenterology Research and Practice
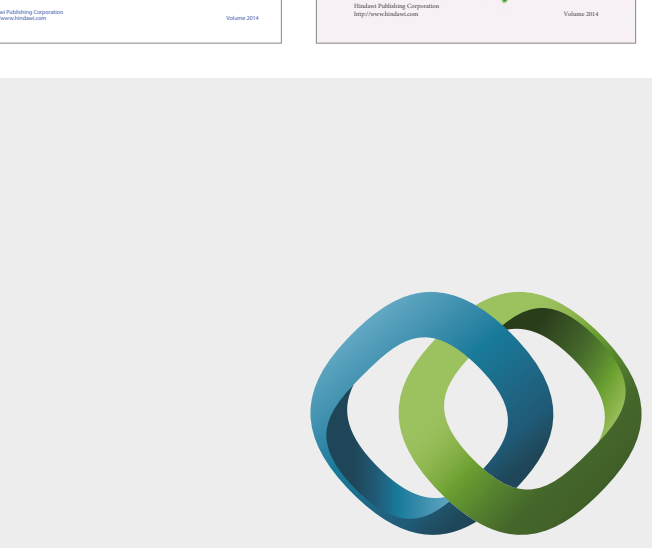

\section{Hindawi}

Submit your manuscripts at

https://www.hindawi.com
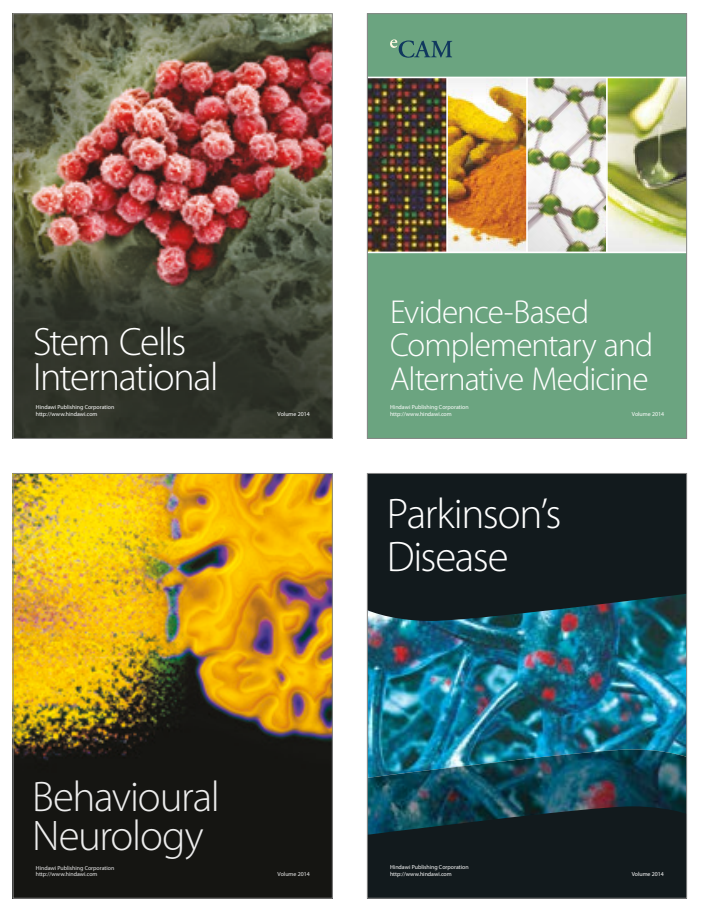
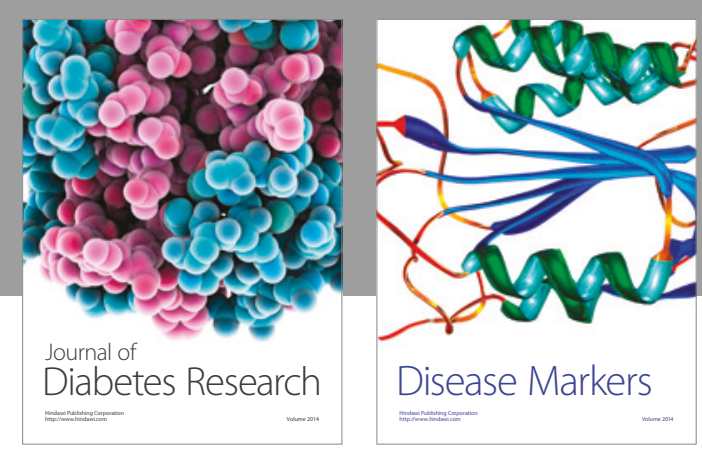

Disease Markers
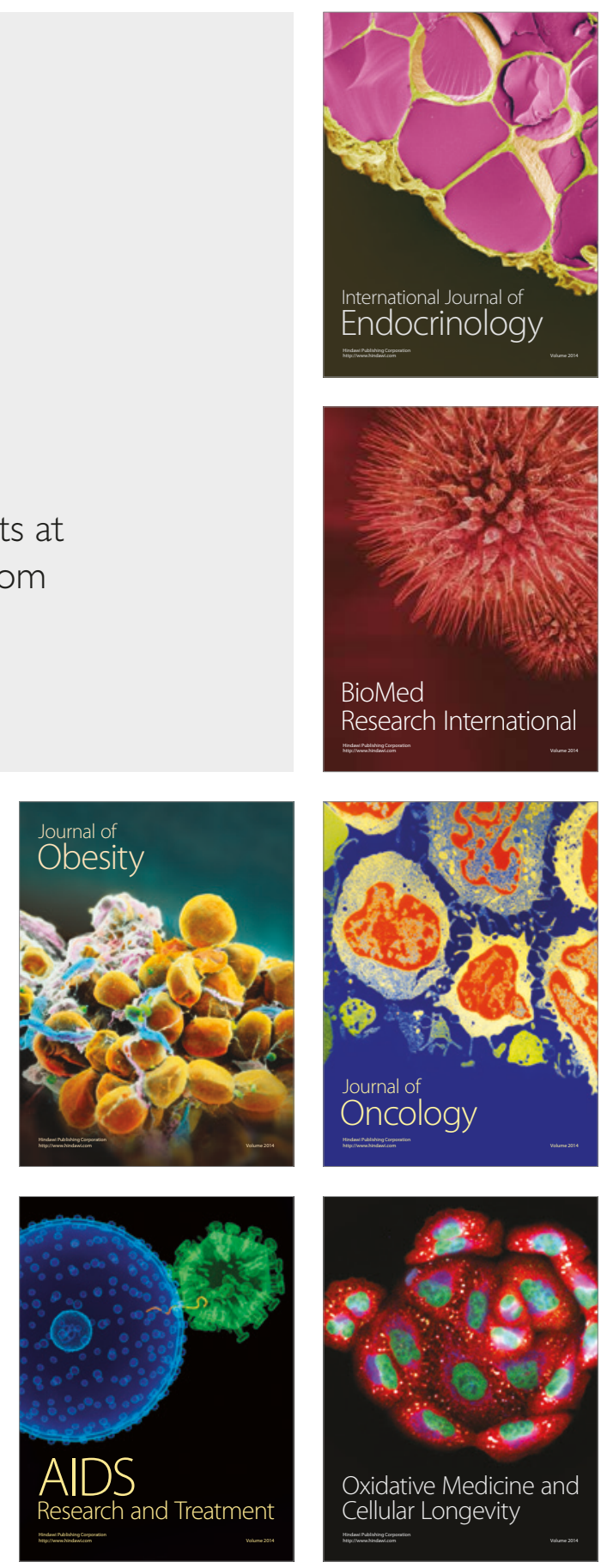